\title{
Dynamic Parallel Adaption for Three Dimensional Unstructured Meshes: Application to Interface Tracking
}

\author{
Youssef Mesri, Walid Zerguine, Hugues Digonnet, \\ Luisa Silva, and Thierry Coupez \\ CIM Group, Center for Material Forming/ MINES ParisTech, Rue Claude Daunesse \\ BP 20706904 Sophia Antipolis cedex France \\ youssef .mesri@ensmp.fr
}

\section{Introduction}

The anisotropic mesh adaption techniques in the last decade have dramatically improved the numerical simulations accuracy of complex problems. An optimal anisotropic mesh adaption consists in refining and coarsening the mesh, by using a metric to specify stretching directions, in order to accurately capture physical anisotropy such as shock waves, contact discontinuities, vortexes, boundary layers and free surfaces. Thus, we propose in this paper, an anisotropic a posteriori error estimator that controls the error due to mesh discretization in all space directions. From the a posteriori error analysis, we obtain an optimal metric (optimal mesh) as a minimum of an error indicator function and for a given number of elements. The optimal metric obtained is used to build an optimal mesh for the given number of elements. Furthermore, solutions for the physical problems illustrated here are often more accurate on adapted meshes than those obtained on globally-refined meshes and at a much lower cost.

The mesh adaption procedure consists in improving iteratively the couple mesh/solution until convergence. This iterative process becomes more expensive when we handle the complex industrial three-dimensional problems.

In this paper, we propose a dynamic parallel mesh adaption on unstructured mesh that is powerful for computing unsteady three-dimensional problems. In this context, we decompose the original 3-dimensional mesh adaption problem into $N_{s}$ smaller subproblems which are solved (i.e., meshed) concurrently using $P$ processors.

Unfortunately, the adaptive solution of unsteady problems causes load imbalance among processors on a parallel machine. This is due to the fact that the computational intensity is not only time dependent, but also varies spatially over the problem domain. However, balancing dynamically the computational load is very difficult. It requires reliable measurements of processor workload and the amount of data transfer, as well as the minimization of interprocessor communication. For this reason, we have built a parallel mesh partitioning/re-partitioning procedure that is extended and customized for FEM computations [7]. The partitioning/ 
re-partitioning scheme allows also to dynamically load balance the workload over heterogeneous architectures [8].

Figure 1 depicts our framework for parallel adaptive flow computation. It consists of a CFD solver and mesh adapter, with a partitioner that redistributes the computational mesh when necessary. The mesh is first partitioned and mapped among the available processors. The flow solver runs for several iterations, updating solution variables. Once a solution is obtained, the mesh adaption procedure is invoked. It targets a re-meshing based on the a posteriori error estimator computed from the flow solution. The old mesh is then locally adapted, generating a new one and followed by the repartitioning procedure to load balance the new workload.

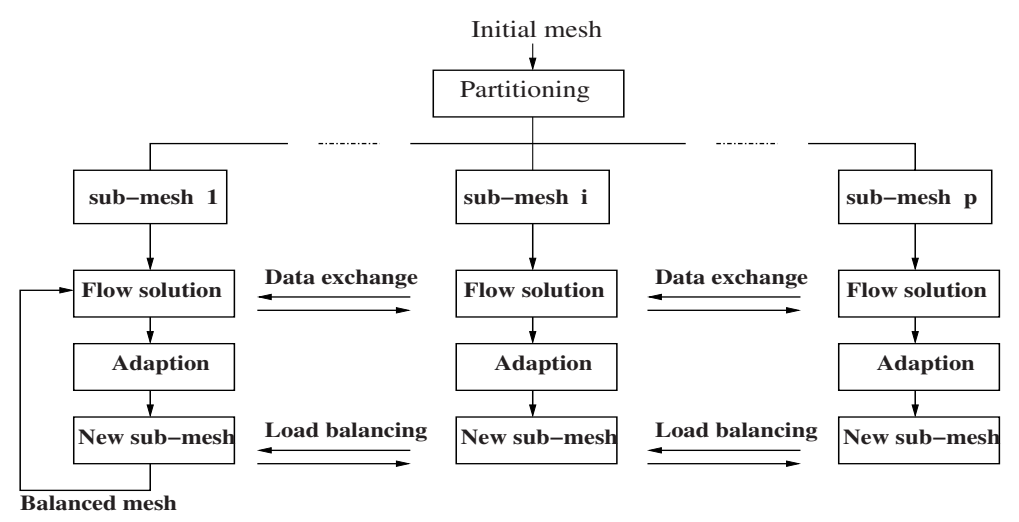

Fig. 1. Overview of our framework for parallel adaptive flow computation

This paper is organized as follows: the first section details the notations and notions that will be used in the sequel. The second section is devoted to the main contribution of this paper, the generalization of Almeida's anisotropic estimator [1] to multi-dimensional unstructured meshes $(n \geq 3)$. The second major contribution consists in the parallel implementation of the mesh adaption procedure, presented in Section 3. In Section 4 we depict numerical results which exhibit the advantage of the combined parallel mesh adaption algorithm and anisotropic error estimator to handle accurately complex CFD problems.

\section{The Optimal Adaptive Mesh Procedure}

\subsection{Notations and Notions}

Given a polygon $\Omega \in R^{d}$, we consider a set of triangulations $\left\{\mathcal{T}_{h}\right\}$. We use the standard subspace of approximation

$$
V_{h}=\left\{v \in H_{0}^{1}(\Omega):\left.v\right|_{T} \in \mathcal{P}_{k}(T)\right\}
$$


where $\mathcal{P}_{k}(T)$ denotes the space of polynomials of degree $k$. We associate, for each node $n_{i}, 1 \leq i \leq \mathcal{N}_{\mathcal{T}_{h}}$ of the triangulation, a basis function $\varphi_{i} \in V_{h}$. For each $i$, we set $S_{i}=\operatorname{supp} \varphi_{i}$.

An a posteriori error estimator for the difference between a given function $u \in W^{2, p}(\Omega)$ and a discrete function $u_{h}$ which is an approximation of $u \in \Omega$ is presented as,

$$
\left\|u-u_{h}\right\|_{L^{p}(\Omega)} \leq C\|u-\Pi u\|_{L^{p}(\Omega)} \leq C\left(\sum_{T \in \mathcal{T}_{h}}\|H(u)(x)\|_{L^{p}(T)}^{p}\right)^{\frac{1}{p}},
$$

where $\Pi: W^{2, p}(\Omega) \rightarrow V_{h}$ is the Clément interpolation operator, $H(u)(x)=D_{2} u(x)\left(x-x_{T}\right)\left(x-x_{T}\right), D_{2} u($.$) is a Hessian operator and x_{T}$ is the barycenter of the element $T$.

The a posteriori error estimator is based on processing the function $u_{h} \in$ $V_{h}$ in order to obtain a better approximation to the Hessian of $u$. We use an approximation instead of the exact Hessian matrix to estimate the $L^{p}$-norm of the error $e=u-u_{h}$.

The process to obtain a recovered Hessian matrix from the function $u_{h}$ is based on a technique to recover the gradient. Zienkiewicz and Zhu [9] used a recovered gradient to estimate the energy norm of the error of the finite element approximation (for more information on recovering first derivatives, see also [10]). Furthermore, Almeida et al. [1] presented an upper bound of the error $\left\|u-u_{h}\right\|_{L^{p}(\Omega)}$ that depends on the recovered Hessian and on the number of elements,

$$
\begin{aligned}
\left\|u-u_{h}\right\|_{L^{p}(\Omega)} \leq C\|u-\Pi u\|_{L^{p}(\Omega)} \leq & C\left(\sum_{T \in \mathcal{T}_{h}}\|H(u)(x)\|_{L^{p}(T)}^{p}\right)^{\frac{1}{p}} \\
& \leq C^{\prime} N_{\mathcal{T}_{h}}^{-\alpha}\left\|H_{R}\left(u_{h}\right)(x)\right\|_{L^{p}(\Omega)}
\end{aligned}
$$

where $\alpha \geq 0$ and $N_{\mathcal{T}_{h}}$ denotes the number of elements of the mesh.

In our work, we use the $L^{p}$-norm of the recovered Hessian as an a posteriori error estimator. To simplify the notation, the recovered Hessian $H_{R}$ will be noted only $H$.

In what concerns re-meshing, our algorithm implies that around an arbitrary point $P$ of the mesh, we try to build equilateral tetrahedrons in the metric defined by the local metric field $\mathcal{M}$, according to a local topological technique. This metric is defined in $R^{d}$ by:

$$
\mathcal{M}(P)=\frac{1}{h_{1}(P)} e_{1} \otimes e_{1}+\cdots+\frac{1}{h_{d}(P)} e_{d} \otimes e_{d}
$$

where $\left(e_{i}\right)_{i=1, d}$ are the eigenvectors of the recovered hessian $H\left(u_{h}(P)\right)$ and $h_{i}(P)$ are the mesh sizes in the $e_{i}$ directions.

\subsection{Anisotropic A Posteriori Error Estimator}

We assume that the function $u_{h} \in V_{h}$ is a good approximation of the function $u$. Hence, we get 


$$
\left\|u-u_{h}\right\|_{L^{p}(\Omega)} \approx C\left\|H\left(u_{h}(x)\right)\left(x-x_{0}\right) \cdot\left(x-x_{0}\right)\right\|_{L^{p}(\Omega)}
$$

where $H\left(u_{h}\right)$ is the recovered Hessian. This shows that the interpolation error in one point $x$ such that $\left|x-x_{0}\right|$ is small enough, is governed by the behavior of the second order derivative in such point. Thus, the interpolation error is not distributed in an isotropic way around point $x_{0}$, i.e., the error depends on direction $x-x_{0}$ and the Hessian matrix value in this point, $H\left(u_{h}(x)\right)$. Therefore, we suggest as in $[15,1,16,14,13,4]$ the use of the expression (5) as a directional local estimator. However, the Hessian matrix is not a metric: it is not positive definite. Therefore, the following tensor is introduced

$$
\mathcal{H}=R \Lambda R^{T}
$$

where $R$ is the orthonormal matrix which corresponds to the eigenvectors $\left(e_{i}\right)_{i=1, d}$ of the Hessian matrix, while $\Lambda=\operatorname{diag}\left(\left|\lambda_{1}\right|, \ldots,\left|\lambda_{d}\right|\right)$ is the diagonal matrix of the absolute values of the eigenvalues of $H\left(u_{h}(x)\right)$. $\mathcal{H}$ can be also written as follows:

$$
\mathcal{H}=R \Lambda R^{T}=\left|\lambda_{1}\right| e_{1} \otimes e_{1}+\cdots+\left|\lambda_{d}\right| e_{d} \otimes e_{d}
$$

Given a mesh discretization $\mathcal{T}_{h}$ of $\Omega$, we define the anisotropic error estimator of the element $T \in \mathcal{T}_{h}$ by

$$
\eta_{T}=\left(\int_{T}\left(\mathcal{H}\left(u_{h}\left(x_{0}\right)\right)\left(x-x_{0}\right) \cdot\left(x-x_{0}\right)\right)^{p} d T\right)^{\frac{1}{p}}
$$

and the global estimator $\eta$ by

$$
\eta=\left(\sum_{T \in \mathcal{T}_{h}} \eta_{T}^{p}\right)^{\frac{1}{p}}
$$

where $x_{0}$ is the barycenter of $T$.

The definition of $\eta_{T}$ can be used to get the following upper bound.

$$
\eta_{T}^{p}=\int_{T}\left(\mathcal{H}\left(u_{h}\left(x_{0}\right)\right)\left(x-x_{0}\right) \cdot\left(x-x_{0}\right)\right)^{p} d T
$$

Substituting (7) in (10) we get,

$$
\begin{aligned}
\eta_{T}^{p} & =\int_{T}\left(\sum_{i=1, d}\left|\lambda_{i}\left(x_{0}\right)\right|\left[e_{i}\left(x_{0}\right) \otimes e_{i}\left(x_{0}\right)\right]\left(x-x_{0}\right) \cdot\left(x-x_{0}\right)\right)^{p} d T \\
& =\int_{T}\left(\sum_{i=1, d}\left|\lambda_{i}\left(x_{0}\right)\right|\left[e_{i}\left(x_{0}\right) \cdot\left(x-x_{0}\right)\right]^{2}\right)^{p} d T
\end{aligned}
$$

If we write $x-x_{0}$ in the eigenvectors basis: $x-x_{0}=\sum_{i=1, d} x_{i} e_{i}$ where $\left|x_{i}\right| \leq h_{i}$, the projection of $x-x_{0}$ on $e_{i}$ direction is $\left[e_{i} \cdot\left(x-x_{0}\right)\right]^{2}=x_{i}^{2} \leq h_{i}^{2}$ and then, 


$$
\eta_{T}^{p} \leq \int_{T}\left(\sum_{i=1, d}\left|\lambda_{i}\left(x_{0}\right)\right| h_{i}^{2}\right)^{p} d T
$$

We notice that an optimal mesh regarding a solution field $u$ is the one that is aligned with this solution. It means that the shape of each element in the mesh is such that the local error in any direction attains the same value. This is equivalent to the fact that the local error in the principal directions of curvature is constant per element i.e.

$$
\left|\lambda_{1}\right| h_{1}=\cdots=\left|\lambda_{d}\right| h_{d}^{2}=\text { cte. }
$$

Then, the stretching of the element $T$ is defined as

$$
s_{1 T}:=\frac{h_{1}}{h_{2}}=\left(\frac{\left|\lambda_{2}\right|}{\left|\lambda_{1}\right|}\right)^{1 / 2}, s_{i T}:=\frac{h_{i}}{h_{i+1}}=\left(\frac{\left|\lambda_{i+1}\right|}{\left|\lambda_{i}\right|}\right)^{1 / 2}, s_{(d-1) T}:=\frac{h_{d-1}}{h_{d}}=\left(\frac{\left|\lambda_{d}\right|}{\left|\lambda_{d-1}\right|}\right)^{1 / 2}
$$

where $h_{i}$ are sorted in the decreasing order, i.e., $h_{1} \geq h_{2} \geq \cdots \geq h_{d}$. Combining (11) and (12) we get

$$
\eta_{T}^{p} \leq|T|\left(d\left|\lambda_{d}\left(x_{0}\right)\right| h_{d}^{2}\right)^{p}
$$

where $|T|$ is the volume of element $T$.

Finally, we obtain the following upper bound of the local error estimator

$$
\eta_{T} \leq d|T|^{\frac{1}{p}}\left|\lambda_{d}\left(x_{0}\right)\right| h_{d}^{2}
$$

This shows that the local estimator is bounded by the maximum of the second order derivative in the barycenter of the element times the square of the longitude in this direction.

We may introduce as the local estimator the above upper bound and is given by

$$
\eta_{T}=d|T|^{\frac{1}{p}}\left|\lambda_{d}\left(x_{0}\right)\right| h_{d}^{2}
$$

In the next section, we define a minimization problem where the functional is the error indicator. The solution of this problem is the metric used to construct an optimal adapted mesh.

\subsection{Optimal Mesh as a Solution of an Optimization Problem}

Let $\mathcal{T}_{h}$ denote the current finite element discretization of the domain $\Omega, u_{h}$ denotes the approximate solution associated to the mesh $\mathcal{T}_{h}, h_{\text {old }}(P)$ describes the local mesh size at node $P$ in the direction of the maximum value of the directional second order derivative at the point $P$. Then, given the number of desired elements $N_{\mathcal{T}_{h}^{\prime}}$ in the new adapted mesh, the optimal mesh adaptive procedure generates a new mesh, $\mathcal{T}_{h}^{\prime}$, such that the new distribution $h_{\text {new }}(P)$, for all $P \in \mathcal{T}_{h}$ minimizes the global estimator error. Hence, the optimal mesh adaptive procedure looks for an optimal mesh as a solution of the following constrained optimization problem 
Y. Mesri et al.

$$
\left\{\begin{array}{l}
\text { Find } h_{T}=\left\{h_{1 T}, \cdots, h_{d T}\right\}, T \in \mathcal{T}_{h} \text { that minimizes the cost } \\
F\left(h_{T}\right)=\sum_{T \in \mathcal{T}_{h}}\left(\eta_{T}\right)^{p} \\
\text { under the constraint } \quad N_{\mathcal{T}_{h}^{\prime}}=C_{0}^{-1} \sum_{T \in \mathcal{T}_{h}} \int_{T} \prod_{i=1}^{d} \frac{1}{h_{i T}} d T
\end{array}\right.
$$

where $C_{0}$ is the volume of a regular tetrahedron. In the constraint equation, the number of desired elements is obtained as follows:

$$
|\Omega|=\sum_{T \in \mathcal{T}_{h}}|T|_{\mathcal{M}}=N_{\mathcal{T}_{h}^{\prime}} C_{0}=\sum_{T \in \mathcal{T}_{h}} \int_{T} \operatorname{det}(\mathcal{M}) d x=\sum_{T \in \mathcal{T}_{h}} \int_{T} \prod_{i=1}^{d} \frac{1}{h_{i T}} d x .
$$

The optimization problem (17) was studied in the case of two dimensional meshes $(d=2)$ in [1]. Here, we generalize the study to three dimensional unstructured meshes. The main difficulty of the $3 \mathrm{D}$ analysis is, that there are different directions of stretching, in opposition to $2 \mathrm{D}$ case where there is only one direction. The following theorem generalizes the anisotropic error estimator proposed in [1] to three dimensional unstructured meshes.

Theorem 1. For $d=3$, the optimization problem (17) has a unique solution and is given by

$$
\left\{\begin{array}{l}
h_{3 T}=\left[\frac{\beta}{\frac{(2 p+3)}{3} C_{1 T}} \int_{T} C_{2 T} d T\right]^{\frac{1}{2(p+3)}} \\
h_{2 T}=s_{2 T} h_{3 T} \\
h_{1 T}=s_{1 T} s_{2 T} h_{3 T}
\end{array}\right.
$$

where

$$
\begin{aligned}
C_{1 T} & =3^{p} C_{0} s_{1 T} s_{2 T}^{2}\left|\lambda_{3}\right|^{p}, \quad C_{2 T}=C_{0}^{-1} \frac{1}{s_{1 T} s_{2 T}^{2}} \quad \text { and } \\
\beta^{\frac{2}{3}(p+3)} & =N_{\mathcal{T}_{h}^{\prime}}^{-1} \sum_{T \in \mathcal{T}_{h}}\left\{\left(\frac{1}{\int_{T} C_{2 T} d T}\right)^{\frac{1}{\frac{2}{3}(p+3)}} \int_{T} C_{2 T}\left[\frac{2 p+3}{3} C_{1 T}\right]^{\frac{1}{\frac{2}{3}(p+3)}} d T\right\} .
\end{aligned}
$$

Proof. Let us consider the following optimization problem:

$$
\left\{\begin{array}{l}
\text { Find } h_{T}=\left\{h_{1 T}, h_{2 T}, h_{3 T}\right\}, T \in \mathcal{T}_{n} \text { such that minimizes the cost function } \\
F\left(h_{T}\right)=\sum_{T \in \mathcal{T}_{h}}|T|\left[3\left|\lambda_{3}\right| h_{3 T}^{2}\right]^{p} \\
\text { under the constraint } \quad N_{\mathcal{T}_{h}^{\prime}}=C_{0}^{-1} \sum_{T \in \mathcal{T}_{h}} \int_{T} \prod_{i=1}^{3} \frac{1}{h_{i T}} d T
\end{array}\right.
$$


Let $s_{1 T}=\frac{h_{1 T}}{h_{2 T}}$ and $s_{2 T}=\frac{h_{2 T}}{h_{3 T}}$ be the two stretching directions of the element $T$ in $R^{3}$. Then, we write $h_{1 T}$ and $h_{2 T}$ as a function of $s_{1 T}, s_{2 T}$ and $h_{3 T}$ :

$$
h_{1 T}=s_{1 T} s_{2 T} h_{3 T}, \quad h_{2 T}=s_{2 T} h_{3 T}
$$

The volume of a tetrahedron $T$ on the metric space can be written as:

$$
|T|_{\mathcal{M}}=|T| \operatorname{det}(\mathcal{M}) \rightarrow|T|=|T|_{\mathcal{M}} \operatorname{det}(\mathcal{M})^{-1}=C_{0} h_{1 T} h_{2 T} h_{3 T} .
$$

where $C_{0}=\frac{\sqrt{2}}{12}$ is the volume of a regular tetrahedron. Thus, with the variable change (21), the 3D optimization problem becomes a 1D optimization problem such as the unique value to look for is $h_{3 T}$

$$
\left\{\begin{array}{l}
\text { Find } h_{3 T}, T \in \mathcal{T}_{n} \text { that minimizes the cost function } \\
F\left(h_{3 T}\right)=C_{0} \sum_{T \in \mathcal{T}_{h}} 3^{p} s_{1 T} s_{2 T}^{2}\left|\lambda_{3}\right|^{p} h_{3 T}^{2 p+3} d T \\
\text { under the constraint } \quad N_{\mathcal{T}_{h}^{\prime}}=C_{0}^{-1} \sum_{T \in \mathcal{T}_{h}} \int_{T} \frac{1}{s_{1 T} s_{2 T}^{2}} \frac{1}{h_{3 T}^{3}} d T
\end{array}\right.
$$

Introducing the following notations

$$
C_{1 T}=3^{p} C_{0} s_{1 T} s_{2 T}^{2}\left|\lambda_{3}\right|^{p}, \quad C_{2 T}=C_{0}^{-1} \frac{1}{s_{1 T} s_{2 T}^{2}} \quad \text { and } \quad \gamma_{T}=\frac{1}{h_{3 T}^{3}} .
$$

The minimization problem can be then written as follows:

$$
\left\{\begin{array}{l}
\text { Find } \gamma_{T}, T \in \mathcal{T}_{n} \text { such that minimizes the cost function } \\
F\left(\gamma_{T}\right)=\sum_{T \in \mathcal{T}_{h}} C_{1 T} \gamma_{T}^{-\frac{2 p+3}{3}} d T \\
\text { under the constraint } \quad N_{\mathcal{T}_{h}^{\prime}}=\sum_{T \in \mathcal{T}_{h}} \int_{T} C_{2 T} \gamma_{T} d T
\end{array}\right.
$$

which is equivalent to the following min-max problem

$$
\min _{\gamma_{T}} \max _{\beta} \mathcal{L}\left(\gamma_{T}, \beta\right)=F\left(\gamma_{T}\right)-\beta\left[N_{\mathcal{T}_{h}^{\prime}}-\sum_{T \in \mathcal{T}_{h}} \int_{T} C_{2 T} \gamma_{T} d T\right]
$$

The solution of this min-max problem is given by

$$
\begin{gathered}
\frac{\partial \mathcal{L}}{\partial \gamma_{T}}=0 \Rightarrow-\frac{2 p+3}{3} C_{1 T} \gamma_{T}^{-\frac{2}{3}(p+3)}+\beta \int_{T} C_{2 T} d T=0 \\
\frac{\partial \mathcal{L}}{\partial \beta}=0 \Rightarrow N_{\mathcal{T}_{h}^{\prime}}-\sum_{T \in \mathcal{T}_{h}} \int_{T} C_{2 T} \gamma_{T} d T=0
\end{gathered}
$$


From (27) we get

$$
\gamma_{T}=\left[\frac{\frac{2 p+3}{3} C_{1 T}}{\beta \int_{T} C_{2 T}}\right]^{\frac{1}{\frac{2}{3}(p+3)}} .
$$

and substituting the expression of $\gamma_{T}$ in (28) we obtain

$$
\beta^{\frac{1}{3}(p+3)}=N_{\mathcal{T}_{h}^{\prime}}^{-1} \sum_{T \in \mathcal{T}_{h}}\left\{\left(\frac{1}{\int_{T} C_{2 T}}\right)^{\frac{1}{\frac{2}{3(p+3)}}} \int_{T} C_{2 T}\left[\frac{2 p+3}{3} C_{1 T}\right]^{\frac{1}{\frac{2}{3}(p+3)}} d T\right\} .
$$

From the definition of the third equation of (24)

$$
h_{3 T}=\left(\frac{1}{\gamma_{T}}\right)^{\frac{1}{3}}=\left[\frac{\beta}{\frac{(2 p+3)}{3} C_{1 T}} \int_{T} C_{2 T} d T\right]^{\frac{1}{2(p+3)}}
$$

We finally get the optimal value of $h_{3 T}$ and then $h_{1 T}$ and $h_{2 T}$ thanks to expression $(21)$.

Remark 1 . We can get the same proof for $d>3$, and that by using a recurrence argument. The generalized form of the theorem can be written as follows:

$$
\left\{\begin{array}{l}
h_{d T}=\left[\frac{\beta}{\frac{(2 p+d)}{d} C_{1 T}} \int_{T} C_{2 T} d T\right]^{\frac{1}{2(p+d)}} \\
h_{i T}=\left(\prod_{k=i}^{d-1} s_{k T}\right) h_{d T}, \quad 1 \leq i \leq d-1
\end{array}\right.
$$

where

$$
\begin{gathered}
C_{1 T}=C_{0} d^{p}\left(\prod_{i=1}^{d-1} s_{i T}^{i}\right)\left|\lambda_{d}\right|^{p}, \quad C_{2 T}=C_{0}^{-1} \frac{1}{\left(\prod_{i=1}^{d-1} s_{i T}^{i}\right)} \text { and } \\
\beta^{\frac{1}{\frac{2}{d(p+d)}}}=N_{\mathcal{T}_{h}^{\prime}}^{-1} \sum_{T \in \mathcal{T}_{h}}\left\{\left(\frac{1}{\int_{T} C_{2 T}}\right)^{\frac{1}{\frac{2}{d(p+d)}}} \int_{T} C_{2 T}\left[\frac{2 p+d}{d} C_{1 T}\right]^{\frac{1}{\frac{2}{d}(p+d)}} d T\right\} .
\end{gathered}
$$

We know now the optimal distribution of the element's shapes and the stretching directions that are given by the eigenvectors of the recovered Hessian as well as the optimal metric that will be used to modify the background mesh. Both are used as an input of the mesh generator tool in order to obtain a new (optimal) mesh. The mesh generator used here and its parallelization will be described in the following section. 


\section{Parallel Mesh Generation}

In this section, we review a parallel mesh generation and adaption procedure based on a topological mesh generator. In the first part (3.1) we describe briefly the sequential mesh generator MTC and then (section 3.2) we illustrate the strategy adopted to parallelize this mesh generator.

\subsection{Mesh Generation}

MTC is a mesh generator developed by Thierry Coupez at the Ecole des Mines de Paris, Center for Material Forming, Sophia Antipolis. It is based on the idea to improve iteratively, an initial unsatisfactory mesh by local improvements.

MTC mesh generator re-meshes the initial mesh iteratively by a local mesh optimization technique. This technique consists in local re-meshing of cavities formed by small clusters of elements in order to increase the "quality" of the elements of the cluster. In the re-meshing process, two principles are enforced:

- The minimal volume, which assures the conformity of the mesh, with no element overlaps: let $\mathcal{T}_{i}(C)$ denote the $i$-th set of elements $T$ filling the local cavity. Following the minimum volume principle we choose as an optimal (possibly not unique) re-triangulation of the cavity the one satisfying

$$
\left.\sum_{T \in \mathcal{T}_{i}(C)} \mid(\text { Volume })(T)\right) \mid \rightarrow \min
$$

where the minimization is done over a small set $i=\{1, \ldots, I\}$ of possible triangulations $\mathcal{T}_{i}(C)$ of elements (Fig. 2 right) connecting the nodes on the border of the cavity, or other nodes like the cavity barycenter, with all boundary faces.
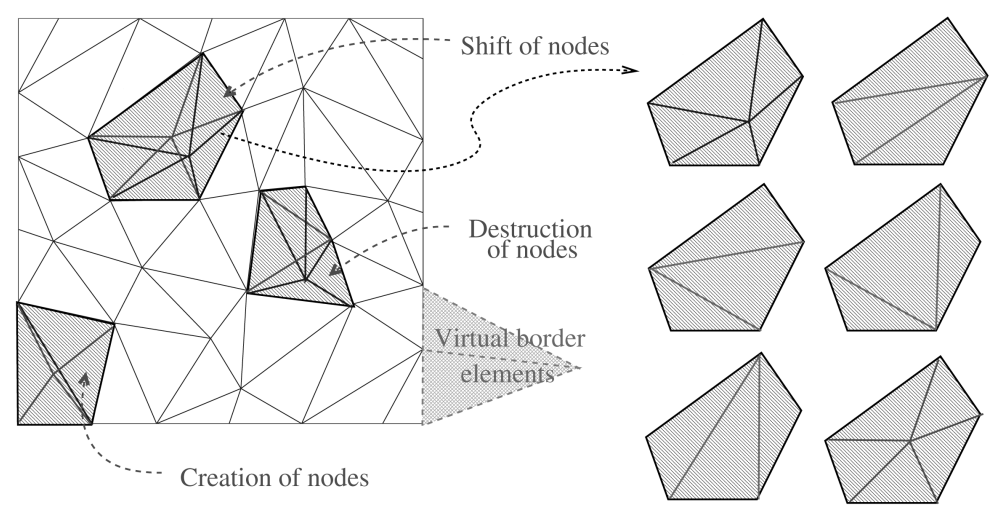

Fig. 2. Local mesh optimization process in MTC 
- The geometrical quality $Q(T)$, which is evaluated for each element. If the minimum of (33) is not unique, this criterion picks among all admissible cavity re-triangulations the one improving the geometrical quality of the mesh by improving the quality of the worst element of the triangulation.

While the former criterion assures the conformity of the mesh, if the initial mesh was conforming, the latter handles improvements of element shape, size, connectivity, etc., depending on the quality function $Q(T)$. Usually, the quality function $Q(T)$ is a function of the geometry of the element $T$ and the prescribed background metric, which give together a measure for the element size and the element form (aspect ratio). For further details see [2].
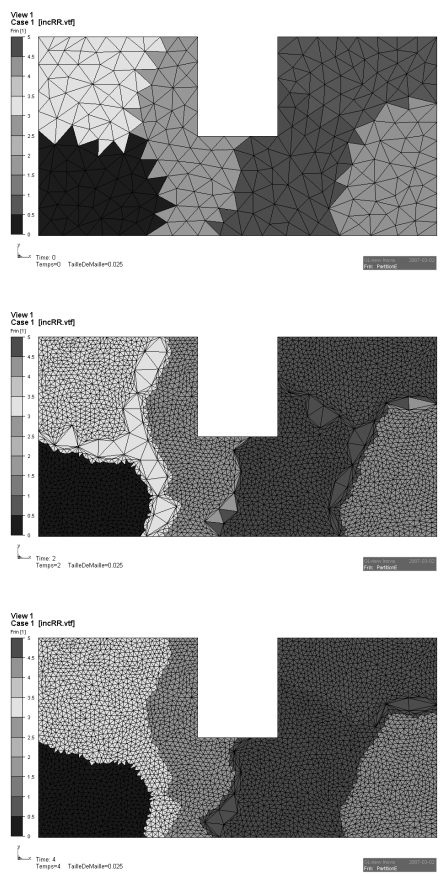
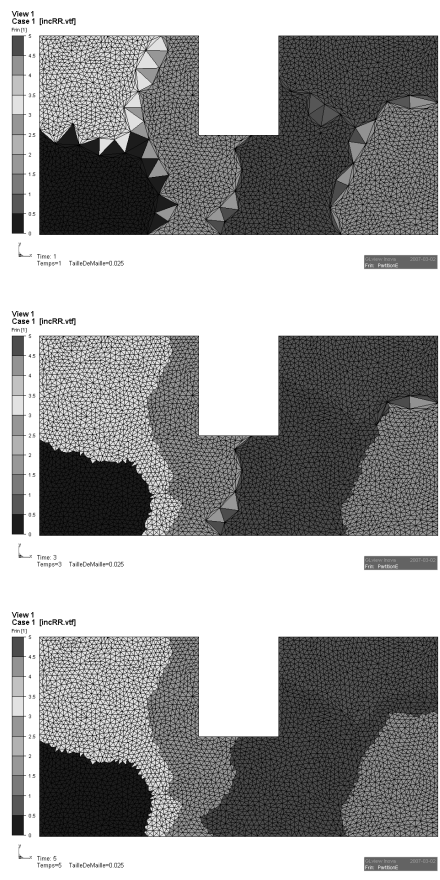

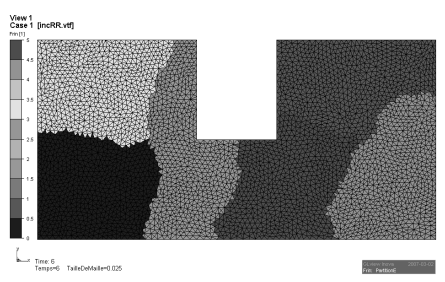

Fig. 3. Illustration of the strategy used to parallelize the mesh generator. From the top to the bottom, we show the successive steps of parallel repartitioning and parallel re-meshing by keeping interfaces unchanged. 


\subsection{Parallel Mesh Adaption Procedure}

The parallelism of the mesh generator MTC is performed by partitioning/ repartitioning the initial mesh into submeshes. The individual submeshes are refined/derefined or adapted to an error estimator by using sequential MTC techniques. In order to achieve mesh conformity and correctness, the interface faces between the submeshes should be subdivided the same way in all submeshes that share them to avoid the non conforming points throughout the process. For this reason, we have chosen to keep interfaces unchanged during the local (inside each subdomain) re-meshing and then to move the interface inside the domain in order to enable re-meshing in a next phase. Figure (3) shows this strategy applied to a simple $2 \mathrm{D}$ mesh with 6 submeshes partitioned onto 6 processors. The partitioning/re-partitioning of a mesh is performed in parallel using a generic graph partitioning that is extended and customized for FEM computations. This software was developed as part of the DRAMA project [7].

\section{Numerical Results}

Using the anisotropic adaption technique described in Section 2 and 3, we study the evolution of the error regarding the number of mesh elements on analytical functions, in two and three dimensions. The global a posteriori error estimator defined in (9) is used here to construct an optimal mesh with respect to the $L^{2}$ error estimator. The global error estimator becomes then:

$$
\eta=\left\{\sum_{T \in \Gamma_{h}}\left(\eta_{T}\right)^{2}\right\}^{1 / 2}
$$

\subsection{D Analytical Cases}

Let us consider the following function

$$
f(x, y)=\tanh (100 y-50+20 \sin (-4 x+4))),(x, y) \in \Omega=[0,1] \times[0,1]
$$

We represent on Fig.4 (left) the analytical function $f$ defined on a square of size $[0,1] \times[0,1]$. The Hessian of the solution is then built by means of a double $L 2$ projection technique. With a number of elements equal to 2000 the minimization process leads to a new size distribution and the metric tensor generates a new adapted mesh described on Fig.4 (right). The final anisotropic mesh obtained is aligned with the analytical solution. We are now interested in the convergence of the $L^{2}$ global estimator regarding the number of elements in the mesh. For this function, a convergence of order 2 is reached Fig. 5.

\subsection{D Analytical Case}

We choose now a more complex function in $3 \mathrm{D}$ in order to check the efficiency of our adaption technique. 


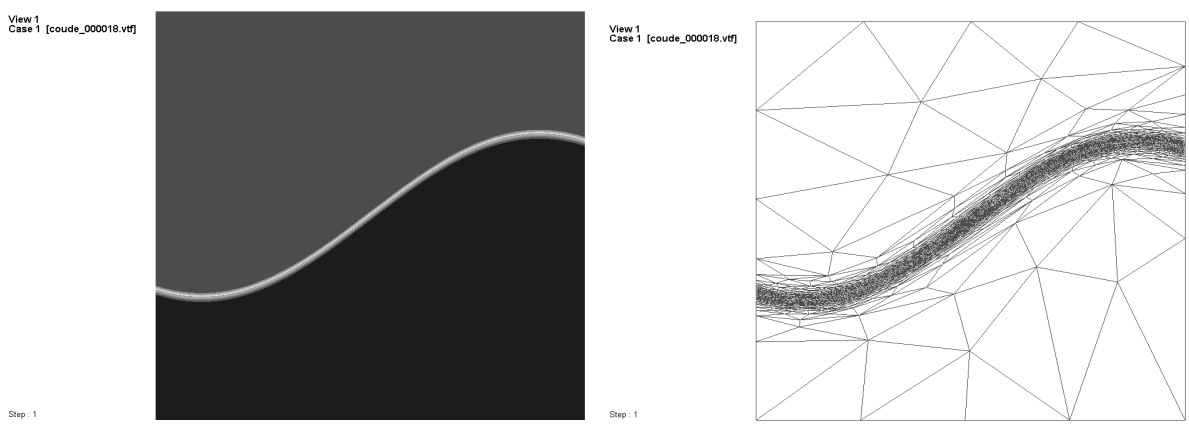

Fig. 4. Visualization of the function $f$ (left) on the adapted mesh (right)

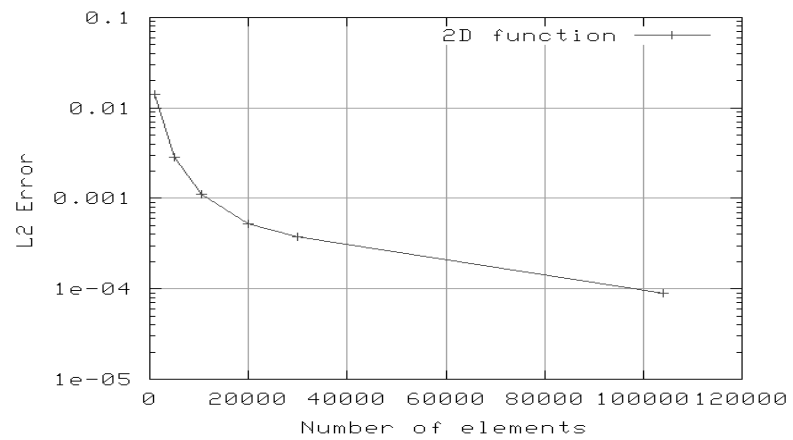

Fig. 5. Evolution of the $L^{2}$ error vs. the number of elements in the mesh for a $2 \mathrm{D}$ analytical test case

$$
h(x, y, z)=\tanh \left((x+1)^{20}(y-0.5)^{9} z\right),(x, y, z) \in \Omega=[0,1] \times[0,1] \times[0,1]
$$

We represent on Fig.6 (left) the analytical function $h$ defined on a cube of size $[0,1] \times[0,1] \times[0,1]$ and, after the mesh adaption strategy, the resulting mesh is depicted in Fig.6 (right). The number of elements targeted is of 100000. As in $2 \mathrm{D}$ case, the mesh emphasizes well with the solution of the analytical function. Moreover, we overcome with success the difficulty occurring in the corners. Indeed, the steep change of directions in the corner regions may be cause of element distortions. We can show that, with this more complex analytical solution and in $3 \mathrm{D}$, we also reach a convergence of order 2 in what concerns the $L 2$ error (Fig.7).

\subsection{Dynamic Adaption Flows}

In this paper, we apply our mesh adaption technique to simulate complex multiphase problems involved in manufacturing processes like Water-Assisted 

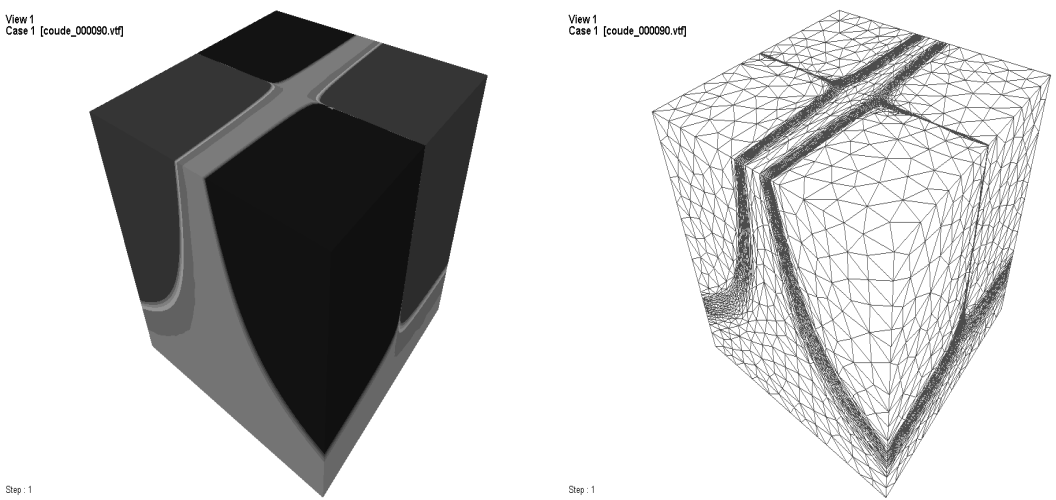

Fig. 6. Visualization of the function $h$ (left) on the adapted mesh (right)

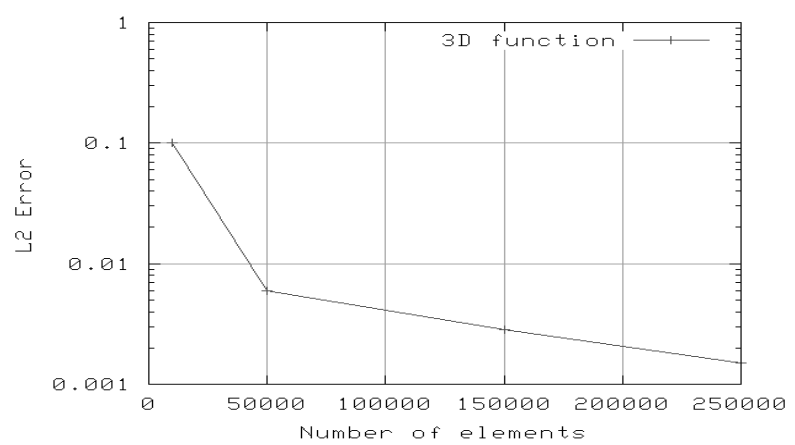

Fig. 7. Evolution of the $L^{2}$ error vs. the number of elements in the mesh for a $3 \mathrm{D}$ analytical test case

Injection Molding (WAIM). The simulation of the process needs an accurate description of the water evolution. The anisotropic strategy aims at improving multiphase flow computations by allowing a better description of physical parameters that characterize strongly heterogeneous flows. Flow equations are solved by considering heterogeneous incompressible Navier-Stokes equations coupled with the heat equation. The water is supposed a newtonian fluid whereas the polymer is considered non newtonian: its viscosity described by WLF and Carreau Yasuda laws, respectively, for the dependency on the temperature field and on the shear rate.

$$
\left\{\begin{array}{l}
\rho \frac{\partial v}{\partial t}+\rho \nabla v \cdot v-\nabla \cdot(2 \eta \varepsilon(v))+\nabla p=\rho g \\
\nabla \cdot v=0 \\
\rho=1_{\text {Swater }} \rho_{\text {water }}+\left(1-1_{\text {Swater }}\right) \rho_{\text {polymer }} \\
\eta=1_{\text {Swater }} \eta_{\text {water }}+\left(1-1_{\text {Swater }}\right) \eta_{\text {polymer }}
\end{array}\right.
$$


where $\rho, \eta, v, p, g$ represent respectively the density, viscosity, velocity, pressure and gravity fields. We denote by $\rho_{\text {water }}, \eta_{\text {water }}, \rho_{\text {polymer }}, \eta_{\text {polymer }}$ the density and viscosity of the water and the polymer. The Navier Stokes equations are solved with a mixed finite element formulation. We consider a linear and continuous velocity enriched spatial discretization $(\mathrm{P} 1+/ \mathrm{P} 1)$.

$$
\left\{\begin{array}{l}
\left.\rho C\left(\frac{\partial T}{\partial t}+\vec{v} \cdot \overrightarrow{\nabla T}\right)-\nabla \cdot(k \overrightarrow{\nabla T})=f \quad \text { on } \Omega \times\right] 0, t_{n}[ \\
\left.T(\vec{x}, t)=T_{\text {imposed }} \text { on } \Omega \times\right] 0, t_{n}[ \\
T\left(\vec{x}, t=t_{0}\right)=T_{0}(\vec{x}) \\
\eta(T, \dot{\gamma}) \text { CarreauYasuda } / W L F) \text { Computation of the thermodependent viscosity }
\end{array}\right.
$$

We follow the polymer/air and water/polymer interfaces with two Level Set functions [12]. The Navier Stokes equations will provide us the velocity and pressure fields and this same velocity will transport the Level Set functions whose zero iso-values represent the interfaces. Therefore, a good description of the interfaces depends on the accurate resolution of the coupled problem. The Level Set strategy applied here is based on a new approach that allows to keep an unitary gradient for the Level Set function without regularizing it periodically with an Hamilton-Jacobi Equation [12].

\section{D dynamic adaption flows}

We apply our adaptive strategy on 2D dynamic flows. We aim to simulate the WAIM process on a $2 \mathrm{D}$ cavity. We impose at the entrance of the cavity a constant

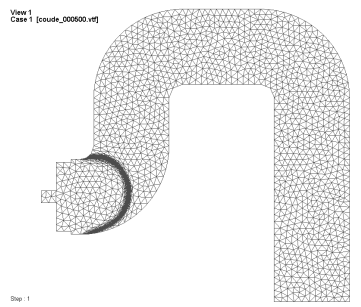

(a)

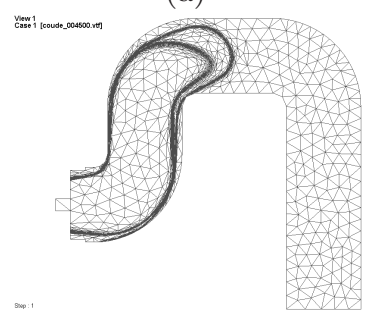

(d)

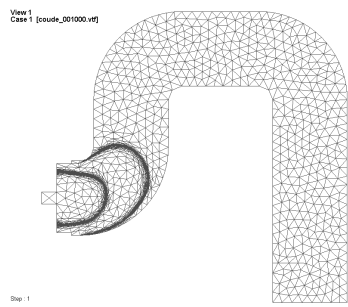

(b)

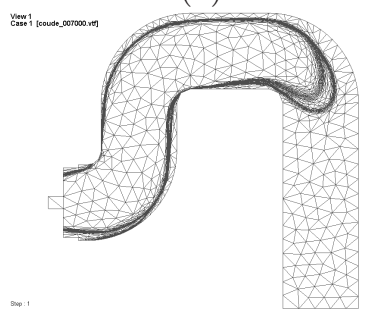

(e)

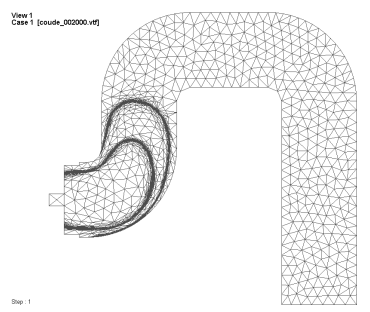

(c)

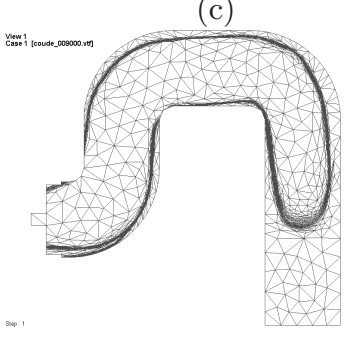

(f)

Fig. 8. Evolution of the polymer/air and water/polymer fronts during the water assisted injection process 


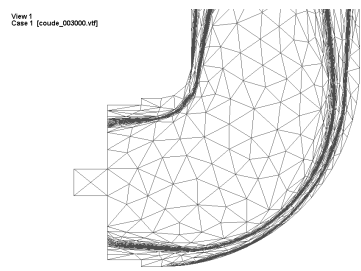

(a)

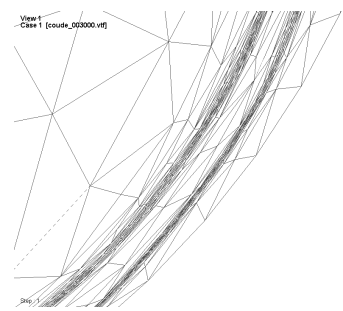

(b)

Fig. 9. Zoom in the mesh

velocity field of $0.1 \mathrm{~m} . \mathrm{s}^{-1}$ in order to be close to the industrial process and to fill the cavity in two seconds. During the first time of the simulation only the polymer is in the cavity Fig.8 (a). Then we change the boundary conditions for the Navier-Stokes equations and we impose a water velocity of $0.2 \mathrm{~m} . \mathrm{s}^{-1}$ at the same entrance. Thus, the water pushes the polymer outside the cavity (Fig.8 from (b) to (f)). The mesh adaption strategy is applied on a reconstructed Level Set function(combination of the polymer/air and water/polymer Level Set functions) and the number of elements aimed is of 20000. Notice that the interface is well captured thanks to anisotropic mesh refinement around the interface, whereas the isotropic region is derefined in order to satisfy the number of elements constraint.

A zoom in the mesh Fig.9 shows us the accuracy of our method.

\section{D industrial Water Assisted Injection Molding case}

The aim of this section is to study the efficiency of our adaptive strategy on an industrial 3D WAIM process Fig.10. So as to achieve our simulation we extend to $3 \mathrm{D}$ space the coupled system described on section 4.3. Few seconds before the water injection the cavity is already filled with the polymer. For this specific simulation the adaption strategy is applied on the water/polymer Level Set function(and not as in the 2D dynamic case on the two Level Set functions).

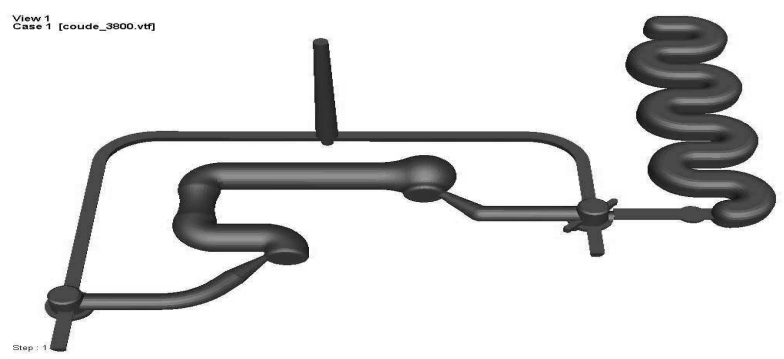

Fig. 10. Water Assisted Injection Molding Cavity 


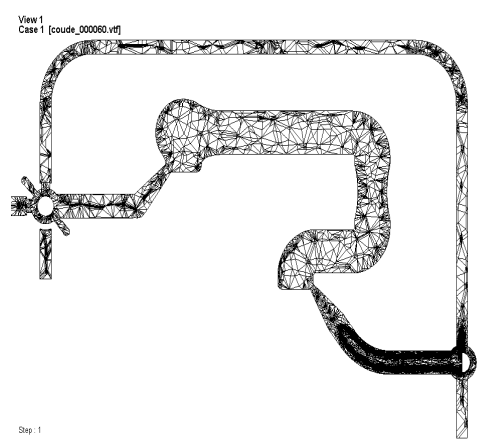

(a)

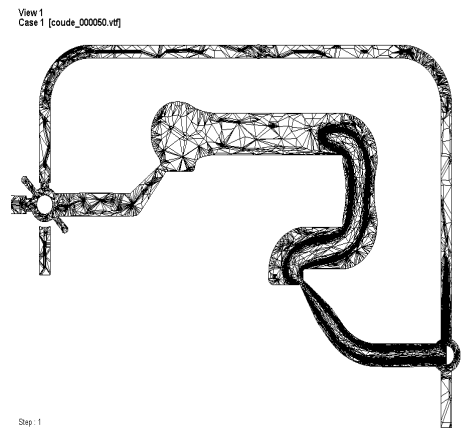

(c)

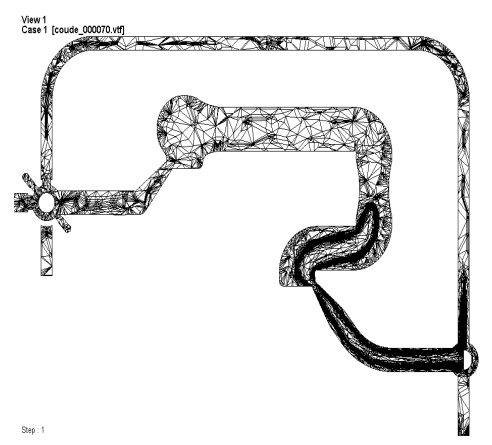

(b)

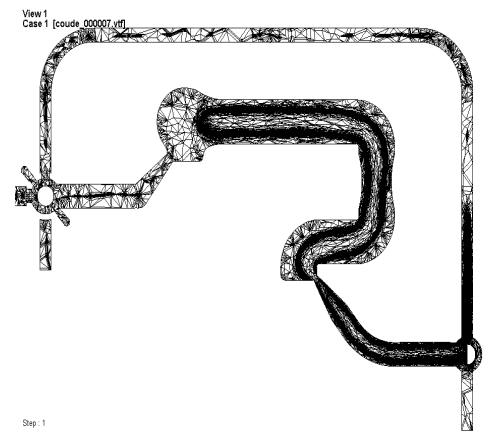

(d)

Fig. 11. Evolution of water/polymer fronts during the water assisted injection process

During the water injection and regarding a certain percentage of injected water a new mesh is generated dynamically.

The simulation is run onto 16 processors and we fix a constraint of 10 millions of elements. A cut of the mesh allows to depict the water tracking inside the cavity at different time of the simulation Fig.11. The mesh follows dynamically the evolution of the water. The adaption process allows an accurate description of the water vein and a considerable gain of CPU time, since, far from the water/polymer front, the size of the elements increases. Therefore the number of unknowns of our system is dramatically reduced.

\section{Conclusions}

We have presented a dynamic parallel mesh adaption procedure. It is based on the definition of an anisotropic a posteriori error estimator, the search of the optimal mesh (metric) that minimizes the error estimator and the use of the serial mesh generator (MTC) in a parallel context. The parallelization strategy consists in balancing dynamically the workflow by repartitioning the mesh after each re-meshing stage. The numerical $2 \mathrm{D}$ and $3 \mathrm{D}$ applications show that the proposed anisotropic error estimator gives an accurate representation of the exact error. 
It was shown also, that the optimal adaptive mesh procedure provides a mesh refinement and element stretching which appropriately captures interfaces for industrial injection polymers problems. Finally, the anisotropic adapted meshes provide a highly accurate solutions that are often better than those obtained on globally-refined meshes and at a much lower cost.

\section{References}

1. Almeida, R., Feijoo, R., Galeao, A., Padra, C., Silva, R.: Adaptive finite element computational fluid dynamics using an anisotropic error estimator. Comput. Methods Appl. Mesh. Engrg. 182, 379-400 (2000)

2. Coupez, T.: Génération de maillage et adaptation de maillage par optimisation locale. In Revue européene des éléments finis 9(4), 403-423 (2002)

3. Coupez, T., Digonnet, H., Ducloux, R.: Parallel meshing and remshing. Appl. Math. Modelling 25(2), 83-98 (2000)

4. Mesri, Y., Alauzet, F., Loseille, A., Hascoet, L., Koobus, B., Dervieux, A.: Continuous metric for computational fluid dynamics. CFD Journal 16(4), 346-355 (2008)

5. Tam, A., Ait-Ali-Yahia, D., Robichaud, M.P., Moore, M., Kozel, V., Habashi, W.G.: Anisotropic mesh adaptation for 3D flows on structured and unstructured grids. Computer Methods in Applied Mechanics and Engineering 189(4), 1205$1230(2000)$

6. Huang, W.: Metric tensors for anisotropic mesh generation. J. Comput. Phys. 204, 633-665 (2005)

7. Basermann, A., Clinchemaillie, J., Coupez, T., Fingberg, J., Digonnet, H., Ducloux, R., Gratien, J.M., Lonsdale, G., Maerten, B., Roose, B., Walshaw, C.: Dynamic load balancing of finite element applications with the DRAMA library. Appl. Math. Modelling 25(2), 83-98 (2000)

8. Mesri, Y., Digonnet, H., Guillard, H.: Mesh Partitioning forParallel Computational Fluid Dynamics Applications On a Grid. In: Finite Volumes for complex applicationsIV, pp. 631-642. Hermes Science Publisher (2005)

9. Zienkiewicz, O.C., Zhu, J.Z.: The superconvergent patch recovery and a posteriori error estimator. Part 1. The recovery technique. Int. J. Numer. Meth. Eng. 33, 1331-1364 (1992)

10. Zienkiewicz, O.C., Zhu, J.Z.: The superconvergent patch recovery and a posteriori error estimator. Part 2. Error estimates and adaptivity. Int. J. Numer. Meth. Eng. 33, 1365-1382 (1992)

11. de Cougny, H., Shephard, M.: Parallel volume meshing using face removals and hierarchical repartitioning. Comp. Meth. Appl. Mech. Engng. 174(3-4), 275-298 (1999)

12. Coupez, T.: Réinitialisation convective et locale des fonctions Level Set pour le mouvement de surfaces et d' interfaces. Journées Activités Universitaires de Mécanique LaRochelle (2006)

13. Lipnikov, K., Vassilevski, Y.: Error bounds for controllable adaptive algorithms based on a Hessian recovery. Computational Mathematics and Mathematical Physics 45(8), 1374-1384

14. Alauzet, F., Loseille, A., Dervieux, A., Frey, P.: Multi-dimensional continuous metric for mesh adaptation. In: Pebay, P. (ed.) Proceedings of 15th International Meshing Roundtable, pp. 163-171. Springer, Heidelberg (2006) 
15. Castro-Diaz, M., Hecht, F., Mohammadi, B., Pironneau, O.: Anisotropic unstructured mesh adaptation for flow simulations. Int. J. Numer. Meth. Fluids 25, 475491 (1997)

16. Dervieux, A., Leservoisier, D., George, P.-L., Coudiere, Y.: About theoretical and practical impact of mesh adaptations on approximation of functions and of solution of PDE. Int. J. Numer. Meth. Fluids 43, 507-516 (2003) 